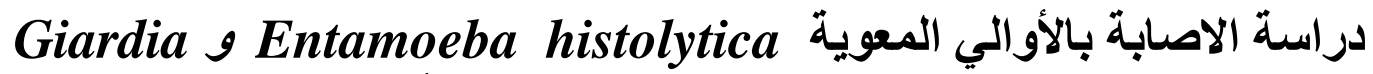

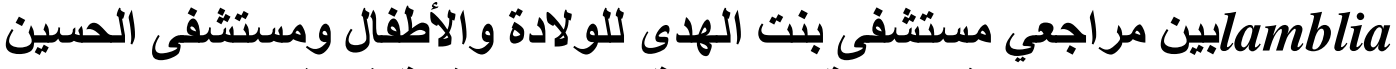

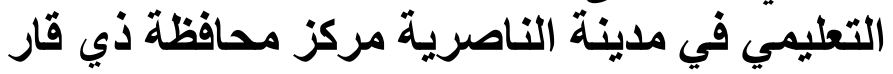

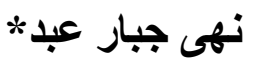

ساهرة كريم فاضل

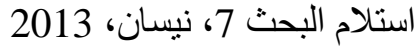

* جامعة ذي قار /كلية العلوم /قسم علوم الحياة |*** وزارة التربية / معهد أعداد المعلمات المعات المعاد

\section{(c) (1) $\Theta$}

قبول النشر 22، كانون الاول، نئان، 2014

NoDerivatives 4.0 International Licens

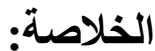

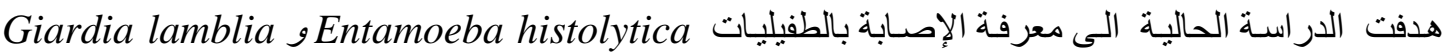

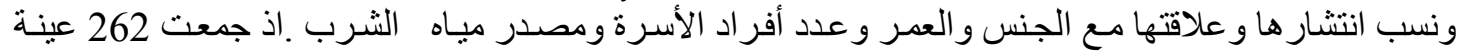

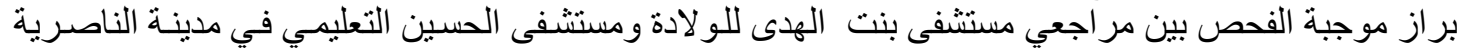

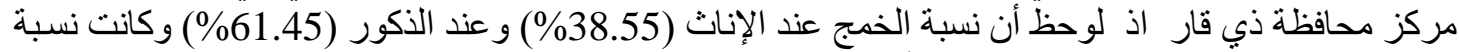

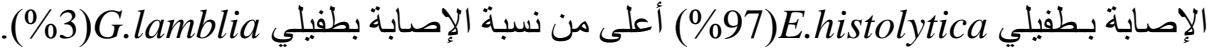

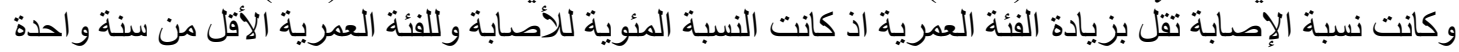

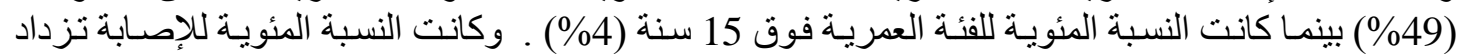

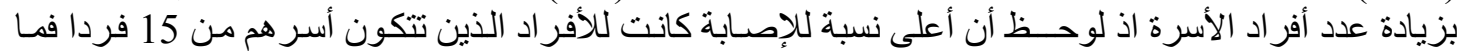
فوق وبنسبة (45\%).

الكلمات المفتاحية:الطفيليات المعوية، جيارديا لامبليا، أميبا الحالة للنسيج.

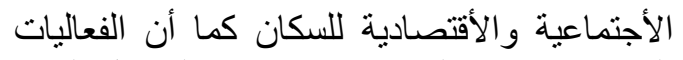

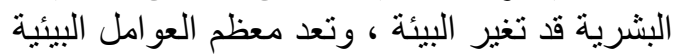

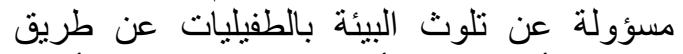

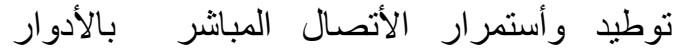

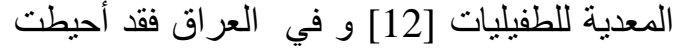

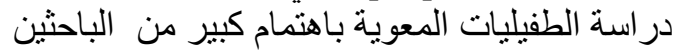

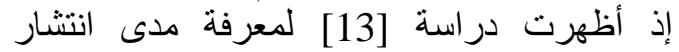

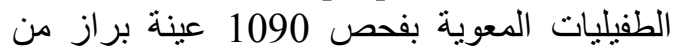
تلامذة المدارس الابتدائية وشملت العينات مركز مدينة الرمادي وخارجها وأسفرت والإنية النتائج عن إنات

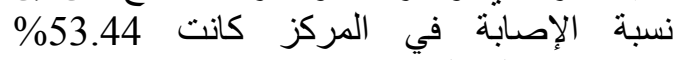
E. وشخصت الطفليات مع نسبها كالاتي:،\%12.29E. coli ،\%14.49 histolytica 7.15\%E. 'T. hominis \% 10.73 A. $6 \% 1.92 \mathrm{H}$. nana 'vermicularis G. lamblia $₫ 0.94$ lumbricoides DientamoebaT. trichiura الـ 60.83 $\% 0.27$ وجد من خلال التحري عن مسبيات الإسهال بين الأطفال الرضع في مستشفيات الرمادي من [14] لإنيات

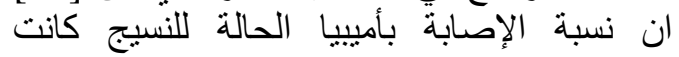
18.1 و والجيارديا لامبليا 4.3\% و والدودة
المقدمة :

الطفليات المعوية المقدة تعد الإصابة بالطفيليات المعوية Intestinal مشكلة صحية عامة خطيرة في أنحاء

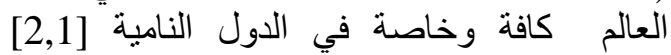
وترتبط الوبائية العالية لها ارتباطا مباشرا بقالة التالة النظافة البيئية] [4,3] وبالفاقة [6,5]

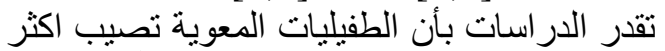

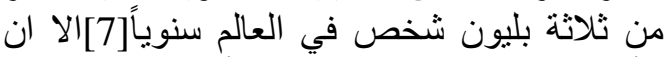

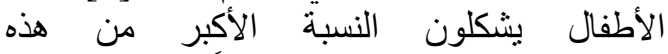

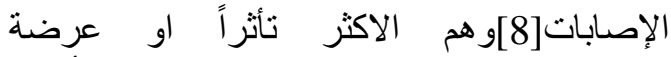
للإِصابة[9] بسبب صغن الإصنابت سنهم ومن ثم قلة أهمية النظافة الصحية [10] بندية أن ازدياد كثافة السكان وضعف الثران

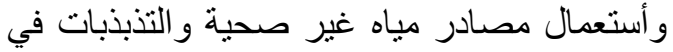
الظروف البيئية تعمل مجتمعة على توفيل توفير مناطق

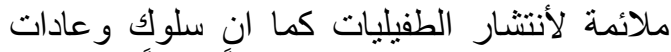
المجموعات السكانية تؤدي أيضاً دوراً مهما في المان

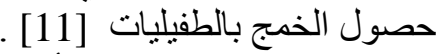

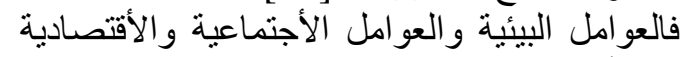

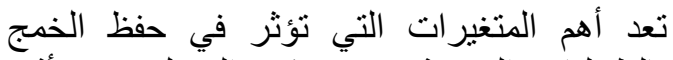

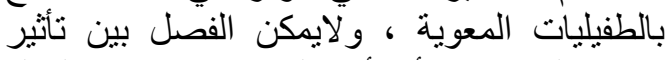
هذين المتغيرين أذ أن البيئة تؤثر في الحئ ليالة 


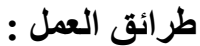

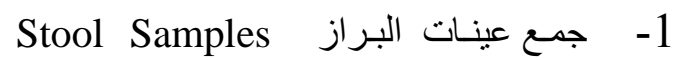
collection

جمعت 262 عينة بر از موجبة الفحص من الاطفال

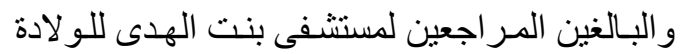

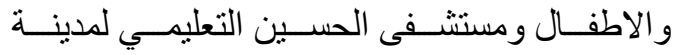

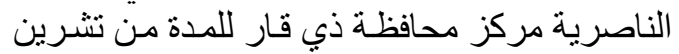
الاول من عام 2011 ولغاية شباط 2012.

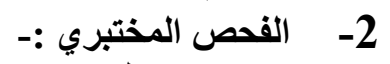

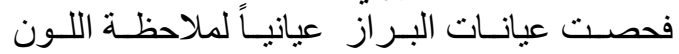

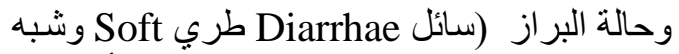

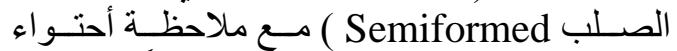

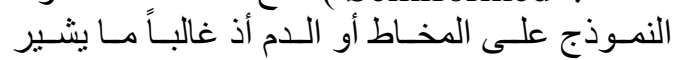

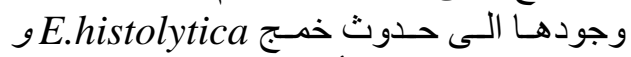
G.lamblia بطريقتين :-

أــــريقـة المســـة المباثــرة المحضَـرة بـالمحلول

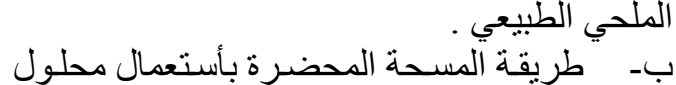
اللوكل - أبودين

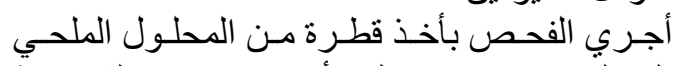

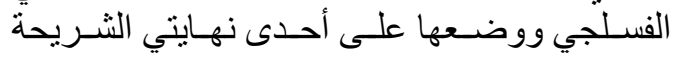

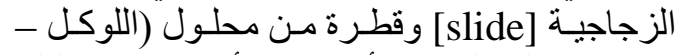

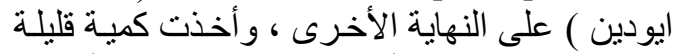

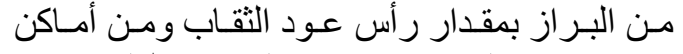

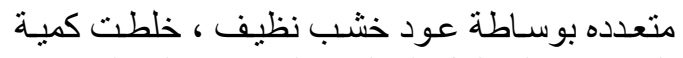
البراز مع المحلول الفسلجي الموجود على على الثريحة

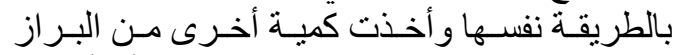

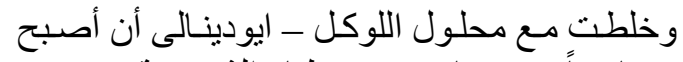

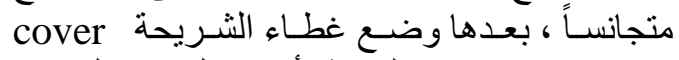

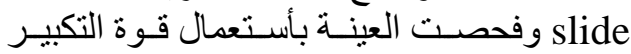
الصغرى ×10 ثم قوة التكبير الكبرى ×40 بأعمان للمجهر

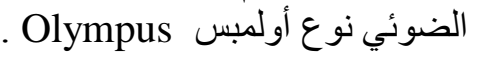

\section{التحليل الإحصائي :}

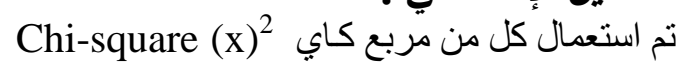
test

أظهرت نتائج فحص 262 نموج بر از موجب

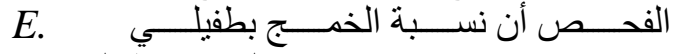
\%97histolytica G. lamblia

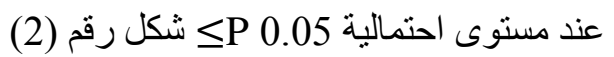

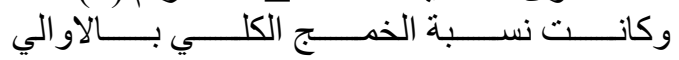
المعويـانة E.histolytica. G. lambia عند الذكور

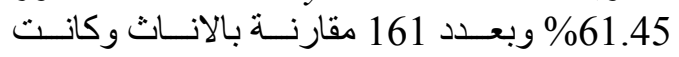

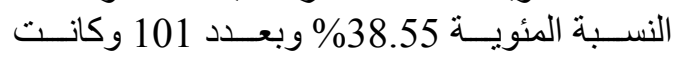

الثريطية القزمةHynenolipsnana0.66\% وفي المعية دراسة عن وبائية الطفيليات المعوية المسبية للأسهال في ديالى سجل [15] وجود فئل من الطفيليات جاءت في مقدمنها الجيارديا لامبليا بنسبة 23\% ثم أميبيا الحالة للنسيج و المشعره البشرية 5\% \% و ؤميبيا القولون 1\% و وأخيراً الدودة الثريطية 0.5\% \%

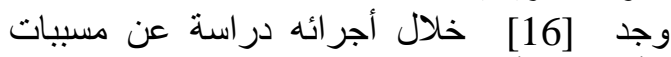

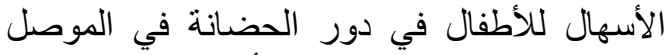
وضمن مناطق مختلفة حصول أصابة كلية مقدار ها لإليا

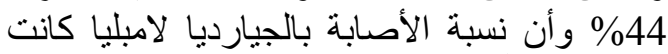
\%38\% فضلاً عن الدودة الثريطية القزمة 20\% و الدودة الدبوسية 15\% و الصفر الخر اطيني 4.8\% \% \%

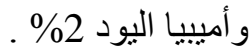
لاحـظ [17] أن نســبة الأصـابة بمسـبيات الأسـهـال

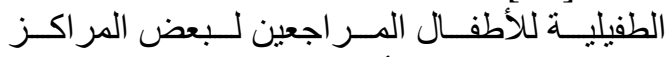

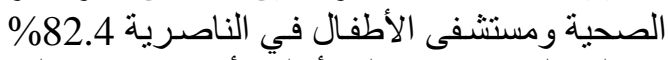

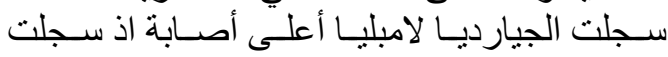

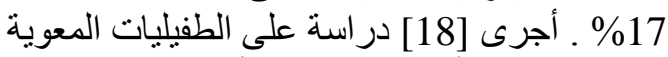

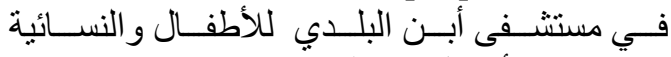
ومستشفى الأطفال في الكاظميـة اذ فحصت الإل 2203

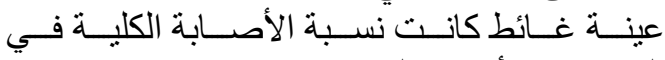

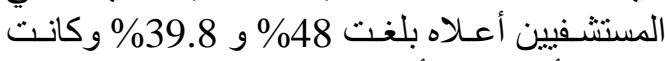

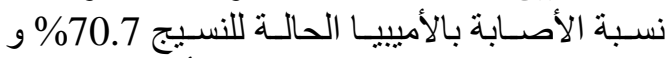

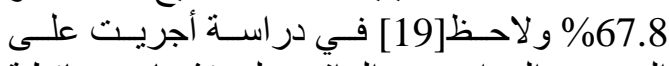

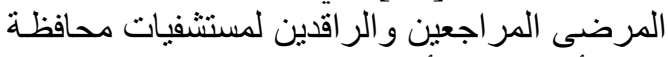

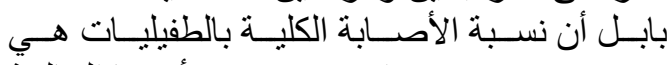

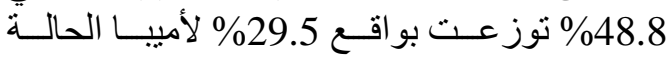

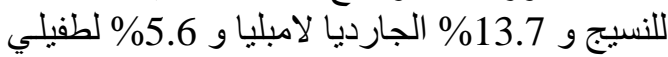
الابو اغ الخبيئة.

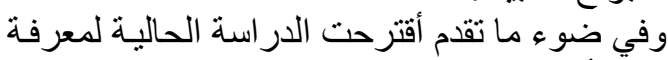
نسب أنتشار الطفيليات المعويـة E.histolytica G. lamblia

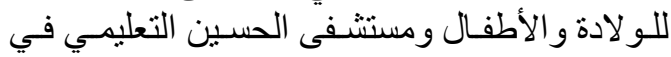

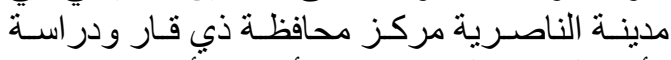

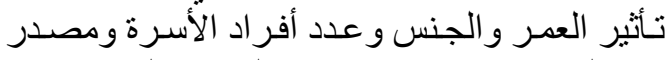

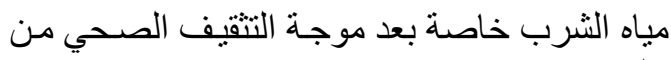
قبل مديرية صحة ذي قارة بدرة

$$
\begin{aligned}
& \text { المواد وطرائق العمل : } \\
& \text { • المواد المستعملة :- }
\end{aligned}
$$

1- المحلول الملحي Normal saline solution

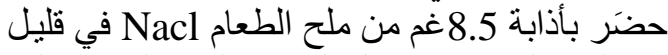

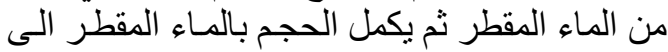
1 1

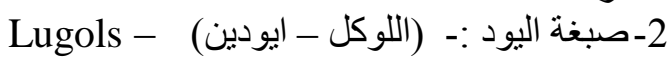
Iodine Stain

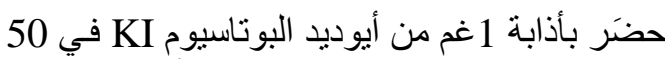

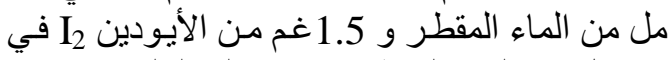
50 مل من الماء المقطر ثم مزج المعلو من لألين . 


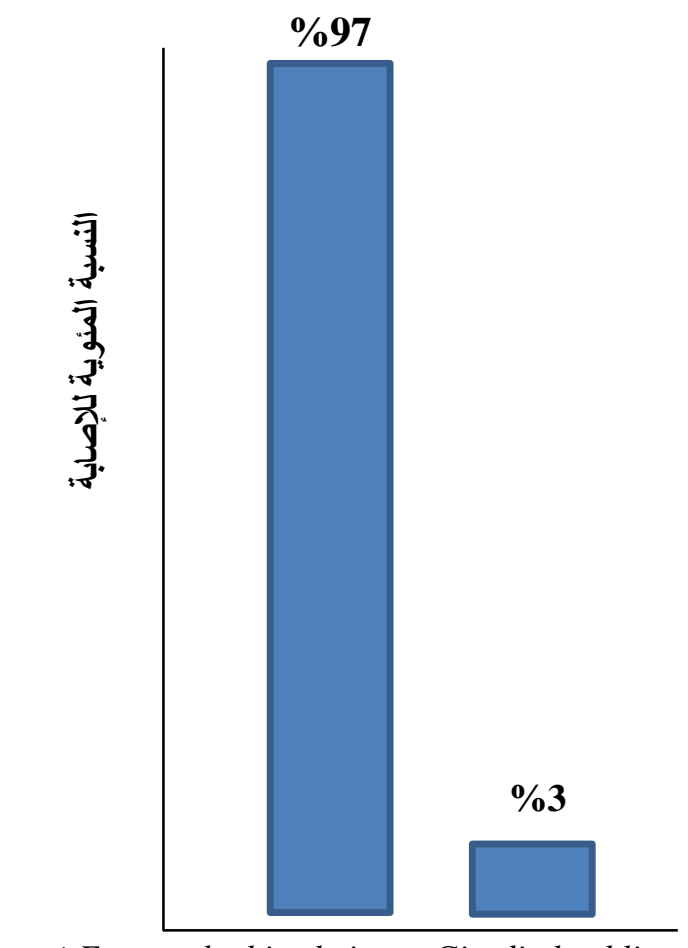

Entamoeba histolytica Giardia lamblia الطفيلي نوع

شكل رقم (2) يمثل العلاقة بين النسبة المئوية للخمج ونوع الطفيلي

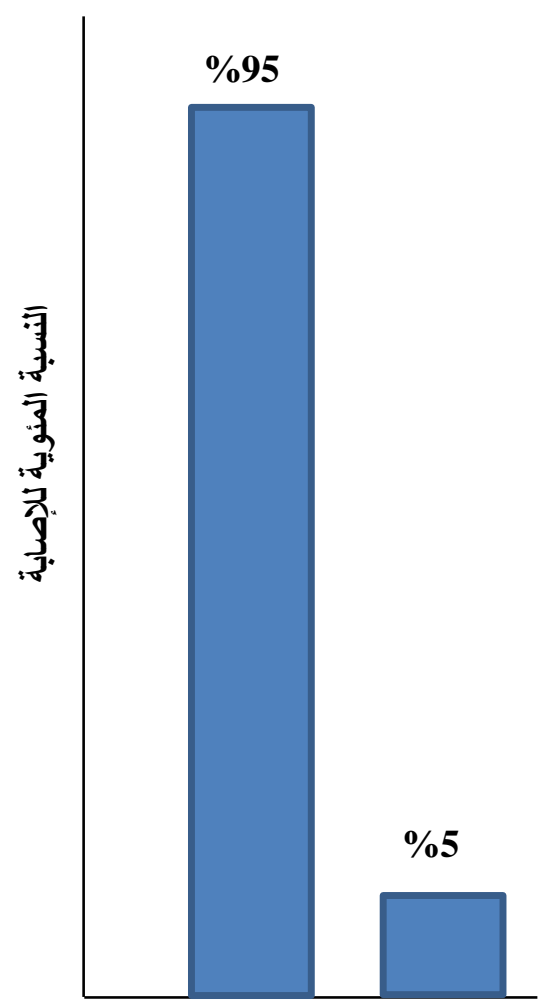

مصدر مياه الثرب ماء معقم ماء حنفية شكل رقم (3) يمثل العلاقة بين النسبة المئوية للخمج ومصدر مياه الثرب الثبة الثن

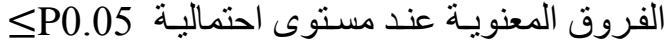

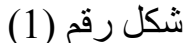

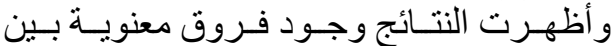

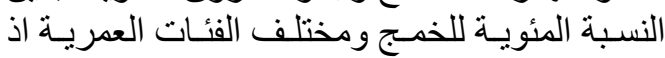

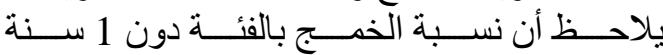
كانت49\% وبعدد (130) مقارنة بالفئة 15 سنة فما

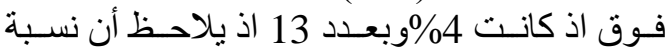

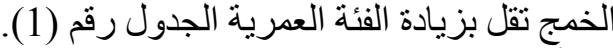

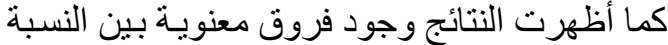

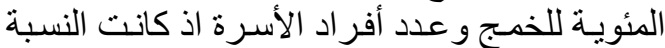

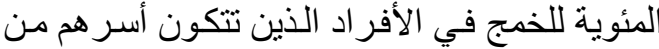

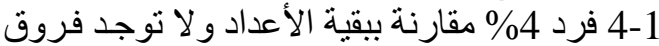

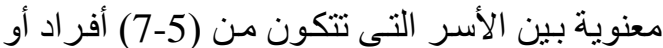

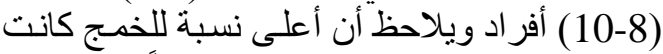

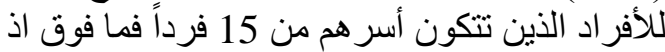

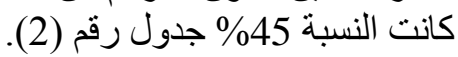

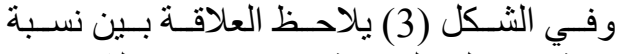

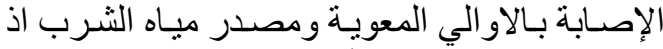

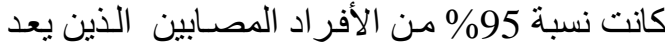

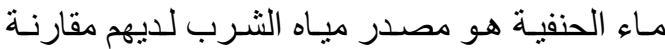

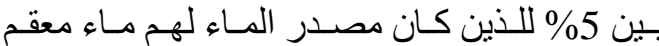

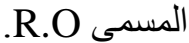

\subsection{5}

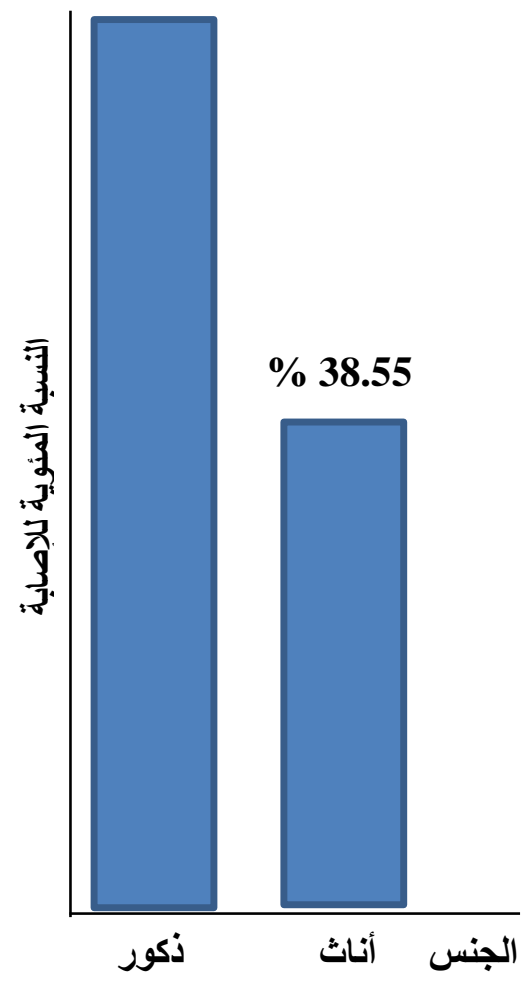

شكل (1) يمثل العلاقة بين النسبة المئوية للخمج

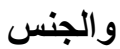


فقد يرجع الى قلـة تلوث (Giardia lamblia)

مياه الثرب التي تعد المصدر الرئيس لأنتقاله اذ اذلي

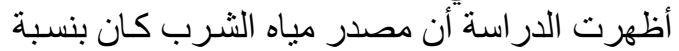

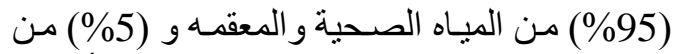

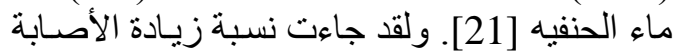
كنها في E.histolytica كل من [25,24,23,22,14

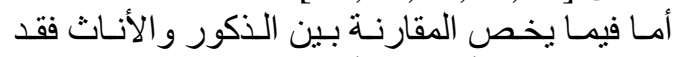
بينت الدر اسـة أن نسبة أصـابة الذكور (61.45\%) والأنـاث (38.55\%) ويعود سبب ذلانك الى الييئة

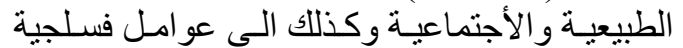

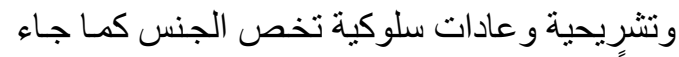
متفقاً مع ما توصل عل اليه [26,19].

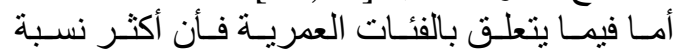

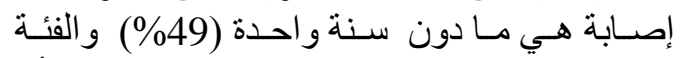

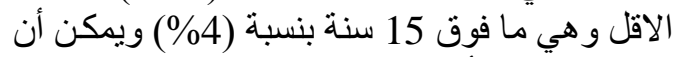

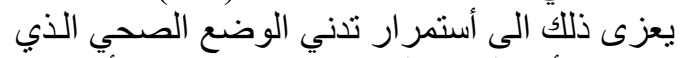

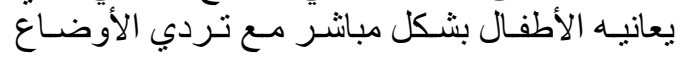

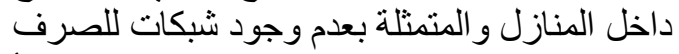

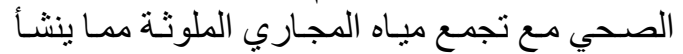

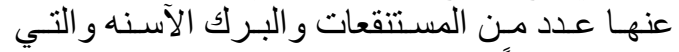

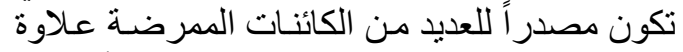

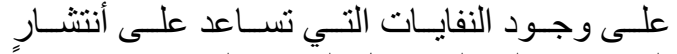

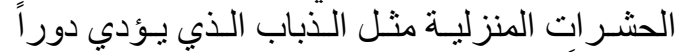

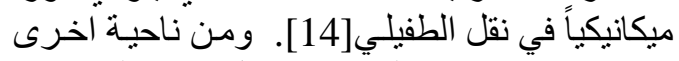

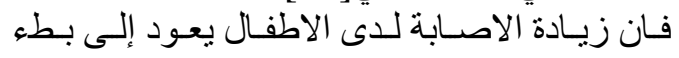

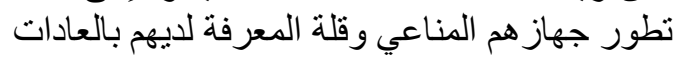
الصحية [27].

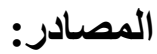

[1] Ogunlesi, T.; Okeniyi, J.; Oseni, S.; O.; Oyelami, O.; Niokanma, F.d an Dedeke O. 2006. Parasitic etiology of childhood diarrhea .Ind. J.paediatr. 73:108-114.

[2] Quihui, L.; Valencai, M. E; Crompton, D. W. T.; Phillips, S.; Hagan, P. and Morales, G. 2006. Role of the employment status and education of mothers in prevalence of intestinal Parasitic infection Mexican rural school children B.M.C. Pub.Health, 6:1-8

[3] Albonico, M.; Crompton, D. W. T and Savioli, L. 1999. Control strategies for human intestinal helminth infection .Adv .Parasitol., 42:276-341.

[4] Montresor, A; Crompton, D. W. T.; Hall, A.; Bundy D.A.P. and
جدول رقم (1) يمثل العلاقة بين العدد والنسبة

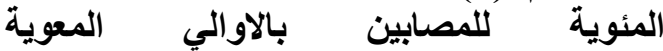
E.histolytica G.lamblia

\begin{tabular}{|c|c|c|}
\hline المئوية & العدد & الفئة العمرية \\
\hline$\% 49$ & 130 & سنة ما دون \\
\hline$\% 22$ & 60 & 5-2 سنة \\
\hline$\% 14$ & 38 & 10-5 سنة \\
\hline$\% 8$ & 21 & 15-11سنة \\
\hline$\% 4$ & 13 & 15 ما فوق \\
\hline
\end{tabular}

جدول رقم (2) يمثل العلاقة بين عدد افراد الاسرة E. والنسبة المئوية للمصابين بالاوالي المعالية histolytica G. lamblia

\begin{tabular}{|c|c|c|}
\hline المئويبة & العدد & النسبة المئوية للإصابة \\
\hline$\% 4$ & 11 & 1-4/فراد \\
\hline$\% 25$ & 66 & 7-5 أفراد \\
\hline$\% 25$ & 66 & 10-8 أفراد \\
\hline$\% 45$ & 119 & 10 ما فوق \\
\hline
\end{tabular}
المناقشة : أظهـرت الدراسـة التـي أجريت على فحس فحس 262

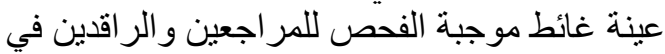

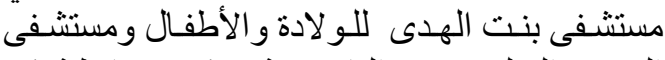

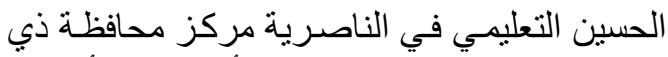

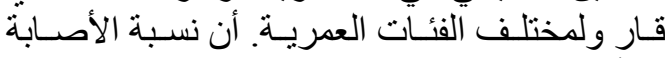

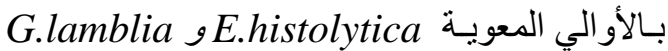

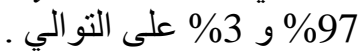

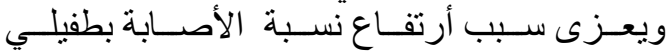
E.histolytica حياتهوسر عة تكاثره و الى سهولة أنتقاله عن طن طريق

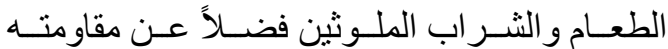

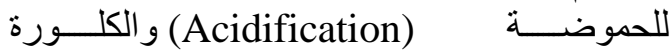
(Chlorination)

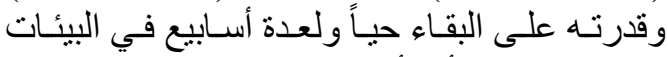

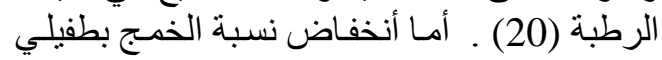


,Coll.Med . AL- NahrainUniv .P. 70.

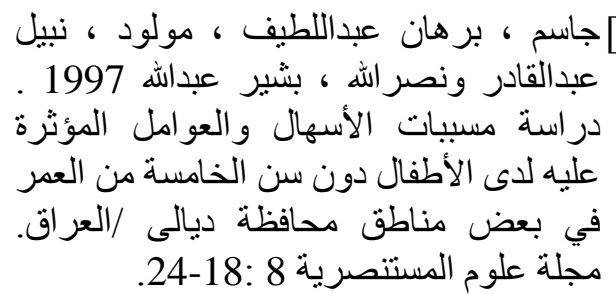

[16] Al-Izzi, N. S. 1998 . Prevalence of intestinal parasitic infection in preschool children in Mosul city. J Fac. Med. Baghdad ,40(4):478480.

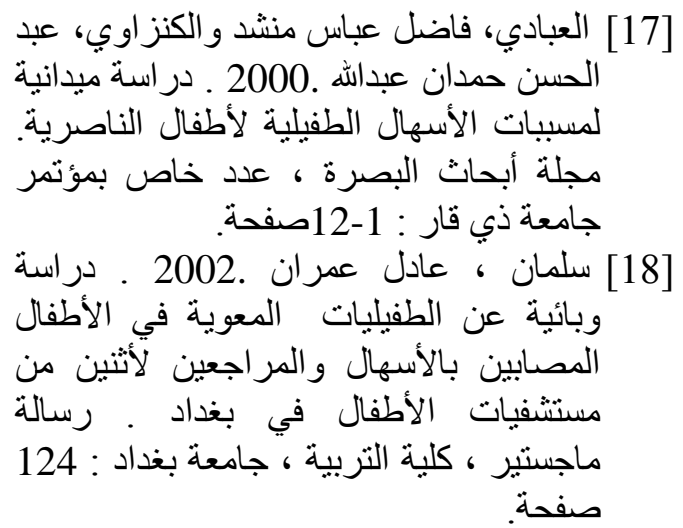

[19] AL-Yassaree, H. F. 2004. Isolation and Identification of three protozoa lenteroparasites .Entamoeba histolytica; Giardia lamblia; and Cr.parvum. M.Sc Thesis, Coll. Sci . Univ .Kufa :P 81

[20] Huston, Ch. D.; R. Haque and W. petri. 1999. Molecular based diagnosis of Entamoeba histolytica infection. Expert. Reviews Molecular medicine Cambridge University Press ISSN: 14623994.

[21] Lechevatiliar, M. W.; W; W.D. Norton and R.G. Lee. 1991. Occurrence of Giardia and Cryptosporidium spp., in surface water supplies, App. Environ .Microbiol ., 57: 2610-2616 .

[22]Niazi, A.D. ; AL-Issa , T.B.; ALkhalissi, A. and Khamis , F. 1983. Ascariasis in Iraq .Bull . E. and Dis. , 10-16 : 69-80.

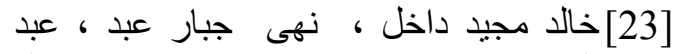
الحسين حبش عواد 2010 ـ دراسة حول دئل
Savioli, L.1998. Guidelines for the Evaluation of Soil Transmitted Helinthisiasis and Schistosomiasis at Community Level .Geneva: W.H.O.

[5] Adamu, H.; Endeshaw, T.; Teka,T.; Kife, A. and petros, B .2006. Prevalence of intestinal parasite .Ethiop J. Health Div .20(1):39-47

[6] Noor Azian, M. Y.; San, Y. M.; Gan, C. C.; Yusri, M. Y.; 2007. rulsyamzawaty, Y.; Zuhaizan, A. H.; Mastawaty, M. N. Norparina, I. and Vythilingam, IPrevalence of intestinal porotozoa in anaborigine community in Pahang, Malaysia. Trop. Biomed., 24:55-62.

[7] Markell, E. K.; john, D. T. and Krotoski, W. A.1999. Markell and Voges Medical Parasitology. $8^{\text {th }}$ ed. Philadelphia: W.B. Saunders.

[8] Fabiana, I. and Carolina, M. 2002. Giardiosis in children - B.M.C. Public health, 2(1) :5-11.

[9] Neouimine, N. I. 1994. Intestinal parasitic infection W.H.O. E.M.R. ,Epidemiol. Bull ., 24

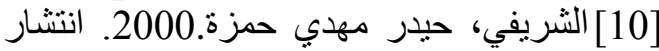

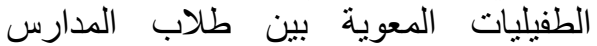
الابتدائية و عمال الاغذية في محافظة التأميم، كابن، العر اق. رسالة ماجستير، كلية التربية، جامعة فئة

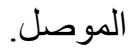

[11] Zeibing. E. A. 1997. Clinical parasitology, $9^{\text {th }}$ Edi, W.B. Saunders company Philadelphia, London, Sydney.

[12] W.H.O. 1984. Prevention and control of amoebiasis Technical Report series 63 (3) : 417-426.

[13] العاني، ميسم خضر 2004. 2004 دراسة مقارنة

للإصابة للطفيليات المعوية بين طلبة اربعة

مدارس ابتدائية في مدينة الرمادي. لإنية رسالة ماجستير ، كلية العلوم ، جامعة الانبار.

[14]Baban, A. A. 1992. Epidemiology of diarrheal diseases in Ramadiarea (Iraq) among infants admitted to pediatric hospital in sixmonths . Dip .Comm .Thesis 
في بعض مناطق محافظة نينوى وتأثثير ها

على مستوى الهيمو غلوبين و أعداد الحمضات

• رسالة ماجستير ، كلية العلوم ، جامعة العاد العات

الموصل : 80صفحة كاحتير

[27]Mengistu, A; Gebre-Selassie, S. and kassa, T. 2007. Prevalence of intestinal parasitic infections prevalence of intestinal parasitic infections among urban dwellers in Southwest. Ethioia. Etamong urban dwellers in Southwest .Ethioia. Ethiop. J. Health Dev.,21(1): 12-17

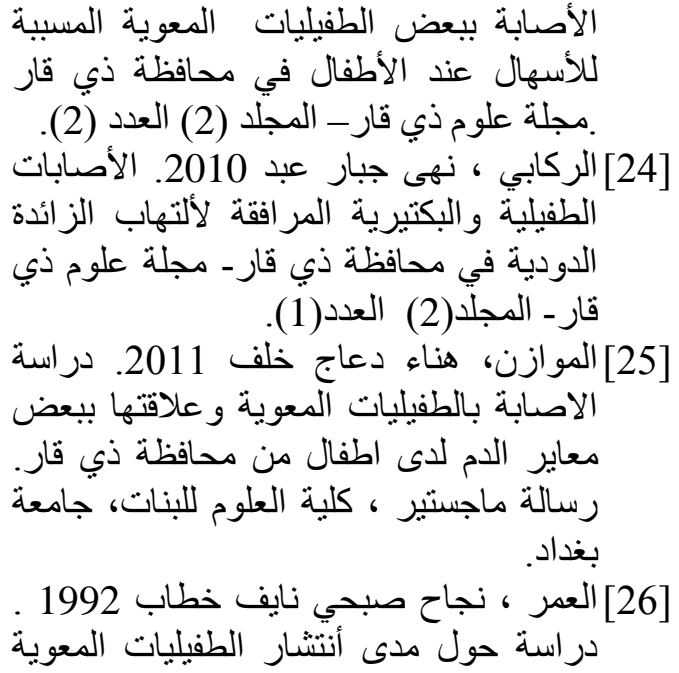

\title{
Study the infection with intestinal protozoa Entamoeba histolytica and Giardia lamblia among Patients who attending Bint Al- Huda for maternity and children hospital and Al hussin hospital in Nassriyia city in Thi-Qar province
}

\author{
Basad A. Al-Aboody* Sahara kareem** \\ NuhaJ.Al-Rekabi* \\ * Department of biology/ faculty of science/ University of Thi-Qar . \\ **Ministry of Education / institute of teachers training.
}

\begin{abstract}
:
The aims of this study the infection with parasites Entamoeba histolytica and Giardia lamblia and prevalence rates and their relationship with gende, age group, the number of family members and source of drinking water among the patients at attending the Bint Al Huda and Al- Hussin Teaching hospital in Nassriyia city in ThiQar province, it was noticed that the percentage of parasitic infection at females was $38.55 \%$ and at males was $61.45 \%$ and the percentage of parasitic infection with Entamoeba histolytica $97 \%$ was higher than the percentage of parasitic infection with Giardia lamblia 3\%,and the reduction of percentage of infection is associated with increasing of the number of family member , the percentage of infection was $49 \%$ at the age group less than one year while it was $4 \%$ at the age group more than 15 year , and the percentage of infection was increasing with number of family members .It was noticed that the higher percentage of infection was $(45 \%)$ at families with members more than 15 person.
\end{abstract}

Key words: Giardia lamblia, Entamoba histolytica, Intestinal Parasites. 